\title{
THE UNION OF TWO HILBERT CUBES MEETING IN A HILBERT CUBE NEED NOT BE A HILBERT CUBE ${ }^{1}$
}

\author{
R. B. SHER
}

AbStract. An example is given to verify the assertion of the title.

For some time there seems to have been a great deal of interest in the following question, which we shall herein answer in the negative: If $X=Q_{1}$ $\cup Q_{2}$, where $Q_{1} \cong Q_{2} \cong Q_{1} \cap Q_{2} \cong Q$, then is $X \cong Q$ ? Here $Q$ denotes the Hilbert cube. The question appears to have first been raised in [1], and has appeared several times since, notably in [3] and [5]. Anderson [1] showed that the answer is yes if $Q_{1} \cap Q_{2}$ is a $Z$-set [2] in each of $Q_{1}$ and $Q_{2}$ and, more recently, Handel [10] has shown that the answer is yes if $Q_{1} \cap Q_{2}$ is a $Z$-set in either $Q_{4}$ or $Q_{2}$.

If should be remarked that our example involves nothing more than an observation regarding already known facts. The first part of the observation is that the construction of Eaton's "dogbone" decomposition [9] of $E^{n}$ can be carried out in $Q$, and that the resulting decomposition space $X$ is not homeomorphic to $Q$. (This has evidently been noted by many others; see, e.g., the remark on p. 153 of [5].) The second part of the observation is that $X$ can be decomposed as $Q_{1} \cup Q_{2}$ where $Q_{1} \cong Q_{2} \cong Q_{1} \cap Q_{2} \cong Q$.

The fact cited as the second part of our observation is a consequence of the following technical

LEMMA. Suppose $M$ is a $Q$-manifold, $C$ a 0 -dimensional compactum, and $F$ : $C \times[0,1] \rightarrow M$ an embedding such that if $0<\delta<1, F(C \times[\delta, 1])$ is a Z-set in $M$. Let $G$ denote the upper semicontinuous decomposition of $M$ whose nondegenerate elements are the members of the set $\{F(\{x\} \times[0,1]) \mid x \in C\}$. Then $M / G \cong M$.

Proof. It suffices to show that $G$ satisfies the following version of the Bing Shrinking Criterion (cf. [6, p. 359, III]): if $U$ is an open set containing the union of the nondegenerate elements of $G$ and $\varepsilon>0$, then there exists a homeomorphism $h$ of $M$ onto itself such that $h(p)=p$ for all $p \in M-U$ and diam $h(g)<\varepsilon$ for all $g \in G$. To verify this, suppose such a $U$ and $\varepsilon$ are

Presented to the Society, March 5, 1976 under the title Some bad embeddings of $Q$ in $Q$; received by the editors November 17, 1975.

AMS (MOS) subject classifications (1970). Primary 57A20.

Key words and phrases. Hilbert cube, dogbone decomposition, Z-set.

'This research was supported by a grant from the UNC-G Research Council. 
given. Choose $\delta$ such that $0<\delta<1$ and $\operatorname{diam} F(\{x\} \times[0,2 \delta])<\varepsilon$ for all $x \in C$. Let $\mathcal{V}$ be an open cover of $U^{\prime}=U-F(C \times\{0\})$ such that (1) if $x \in C$, then there exists $V \in \mathcal{V}$ such that $F(\{x\} \times[\delta, 1]) \subset V$, and (2) if $h^{\prime}$ is a homeomorphism of $U^{\prime}$ onto itself which is $\mathrm{St}^{5}(\mathfrak{V})$-close to the identity, then $h^{\prime}$ extends, via the identity on $M-U^{\prime}$, to a homeomorphism of $M$ onto itself. Let $A=C \times(0,1]$. Define $f^{\prime}: A \rightarrow U^{\prime}$ by $f^{\prime}(x, t)=F(x, t)$ for all $(x, t) \in A$ and $g^{\prime}: A \rightarrow U^{\prime}$ by

$$
g^{\prime}(x, t)= \begin{cases}F(x, t) & \text { if } x \in C \text { and } 0<t \leqslant \delta, \\ F\left(x, \frac{\delta t+\delta-2 \delta^{2}}{1-\delta}\right) & \text { if } x \in C \text { and } \delta \leqslant t \leqslant 1 .\end{cases}
$$

Then $f^{\prime}(A)$ and $g^{\prime}(A)$ are $Z$-sets in $U^{\prime}$ and $f^{\prime} \simeq g_{p}$ via a proper homotopy limited by $\mathcal{T}$. It follows from Theorem 6.1 of [4] that there exists an invertible ambient isotopy $H: U^{\prime} \times[0,1] \rightarrow U^{\prime}$ such that $H_{0}=\mathrm{id}, H_{1} f^{\prime}=g^{\prime}$, and $H$ is limited by $\mathrm{St}^{5}(\mathcal{V})$. Extending $H_{1}$ to $M$ via the identity on $M-U^{\prime}$, we obtain a homeomorphism $h$ of $M$ onto itself satisfying the requirements of the shrinking criterion.

The EXAMPLE. In [9], Eaton constructs a "dogbone" decomposition of $E^{n}$, $n \geqslant 3$, using a "ramified" version of Blankinship's construction of a wild Cantor set in $E^{n}$ [7]. Wong [11] modified Blankinship's construction to obtain a Cantor set $K \subset Q$ such that $\pi_{1}(Q-K)$ is nontrivial. Using Wong's description, it is easy to see how to ramify the construction of $K$ as in [9] to obtain a Cantor set $L$ in $Q$ having the necessary complications for the construction of [9] to be carried out. (The formalities of the ramification process are detailed in $[8, \S 4]$.) In essence this construction can be thought of as identifying a Cantor set of Cantor sets, each embedded in $Q$ as $K$ is embedded in $Q$.

Now, $Q-L$ contains a $Z$-set homeomorphic to $Q$ and, as previously mentioned, gluing two copies of $Q$ along such a set yields a copy of $Q$. Identifying the resulting space with $Q$, we see that we may write $Q=Q^{\prime} \cup$ $Q^{\prime \prime}$, where $Q^{\prime \prime \prime}=Q^{\prime} \cap Q^{\prime \prime} \cong Q$ is a $Z$-set in each of $Q^{\prime}$ and $Q^{\prime \prime}$, and where there exist homeomorphisms $h^{\prime}: Q \rightarrow Q^{\prime}$ and $h^{\prime \prime}: Q \rightarrow Q^{\prime \prime}$ such that $h^{\prime}(L) \cap$ $Q^{\prime \prime \prime}=\varnothing=h^{\prime \prime}(L) \cap Q^{\prime \prime \prime}$. We may further assume that the manifolds of the "special defining sequences" [8] used in defining $h^{\prime}(L)$ and $h^{\prime \prime}(L)$ fail to intersect $Q^{\prime \prime \prime}$.

The reader who is familiar with [9] will now have no difficulty in seeing how to obtain an embedding $H: C \times[0,1] \rightarrow Q$, where $C$ is a Cantor set, such that

(1) for each $x \in C, H(\{x\} \times[0,1])$ is a $Z$-set in $Q$,

(2) $H(C \times\{0\})=h^{\prime}(L)$ and $H(C \times\{1\})=h^{\prime \prime}(L)$,

(3) $Q^{\prime \prime \prime} \cap H(C \times[0,1])=H\left(C \times\left\{\frac{1}{2}\right\}\right)$,

(4) if $0<\delta<\frac{1}{2}$, then $H\left(C \times\left[\delta, \frac{1}{2}\right]\right)$ is a $Z$-set in $Q^{\prime}$ and

$$
H\left(C \times\left[\frac{1}{2}, 1-\delta\right]\right)
$$


is a $Z$-set in $Q^{\prime \prime}$, and

(5) if $G$ is the upper semicontinuous decomposition of $Q$ whose nondegenerate elements are the members of the set $\{H(\{x\} \times[0,1]) \mid x \in C\}$, then $Q / G \simeq Q$.

Let $X=Q / G$ and let $\Pi: Q \rightarrow X$ denote the natural projection. Letting $Q_{1}=\Pi\left(Q^{\prime}\right)$ and $Q_{2}=\Pi\left(Q^{\prime \prime}\right)$, it follows from the Lemma and condition (4) above that $Q_{1} \cong Q \cong Q_{2}$. But $X=Q_{1} \cup Q_{2}$ and, by condition (3), $Q_{1} \cap Q_{2}$ $=\Pi\left(Q^{\prime \prime \prime}\right) \cong Q$, so $X$ provides the promised example.

We note that the above construction can be carried out in such a way that $H\left(C \times\left\{\frac{1}{2}\right\}\right)=C^{\prime}$ is a $Z$-set in $Q^{\prime \prime \prime}$. Then there is a $(f-d)$ cap set in $Q^{\prime \prime \prime}-C^{\prime}$, and it follows easily that there is a $(f-d)$ cap set in $Q_{1} \cap Q_{2}$ which is a $\sigma-Z$-set in each of $Q_{1}$ and $Q_{2}$. On the other hand, we could arrange things so that $H\left(C \times\left\{\frac{1}{2}\right\}\right)=C^{\prime \prime}$ is embedded in $Q^{\prime \prime \prime}$ as $L$ is embedded in $Q$, with $H\left(C \times\left[0, \frac{1}{2}\right]\right)$ providing a "mismatch" between $h^{\prime}(L)$ and $C$ ". In this case, there will be a disk $D$ in $Q_{1} \cap Q_{2}$ such that any disk "close" to $D$ in $Q_{1} \cap Q_{2}$ contains a Cantor set whose complement in $Q_{1}$ fails to be simply connected (the argument for this is contained in [8]). It follows that in this case there does not exist a $(f-d)$ cap set in $Q_{1} \cap Q_{2}$ which is a $\sigma$-Z-set in $Q_{1}$.

AdDed IN Proof. J. Quinn and R. Y. T. Wong have recently shown that the union of two Keller cubes (compact convex infinite dimensional subsets of $l_{2}$ ) meeting in a Keller cube is homeomorphic to $Q$.

\section{REFERENCES}

1. R. D. Anderson, Topological properties of the Hilbert cube and the infinite product of open intervals, Trans. Amer. Math. Soc. 126 (1967), 200-216. MR 34 \# 5045.

2. , On topological infinite deficiency, Michigan Math. J. 14 (1967), 365-383. MR 35 \#4893.

3. R. D. Anderson and R. H. Bing, A complete elementary proof that Hilbert space is homeomorphic to the countable infinite product of lines, Bull. Amer. Math. Soc. 74 (1968), 771-792. MR 37 \# 5847.

4. R. D. Anderson and T. A. Chapman, Extending homeomorphisms to Hilbert cube manifolds, Pacific J. Math. 38 (1971), 281-293. MR 47 \#7499.

5. R. D. Anderson and Nelly Kroonenberg, Open problems in infinite-dimensional topology, Math. Centre Tracts, no. 52, Mathematisch Centrum, Amsterdam, 1974, pp. 141-175.

6. R. H. Bing, A homeomorphism between the 3-sphere and the sum of two solid horned spheres, Ann. of Math. (2) 56 (1952), 354-362. MR 14, 192.

7. William A. Blankinship, Generalization of a construction of Antoine, Ann. of Math. (2) 53 (1951), 276-297. MR 12, 730.

8. Robert J. Daverman, On the scarcity of tame disks in certain wild cells, Fund. Math. 79 (1973), 63-77. MR 48 \# 5085.

9. W. T. Eaton, A generalization of the dog bone space to $E^{n}$, Proc. Amer. Math. Soc. 39 (1973), 379-387. MR 48 \# 1238.

10. Mike Handel, On certain sums of Hilbert cubes (to appear).

11. Raymond Y. T. Wong, A wild Cantor set in the Hilbert cube, Pacific J. Math. 24 (1968), 189-193. MR 36 \#4539.

Department of Mathematics, University of North Carolina at Greensboro, GreensBORO, NORTH CAROLINA 27412 